\begin{tabular}{ccc}
\hline & International Journal of Engineering \&Technology, $7(3.12)(2018) 228-231$ \\
SPC & International Journal of Engineering \& Technology \\
\hline
\end{tabular}

\title{
Hard Decision Decoding Performance Improved Using Turbo Product Codes
}

\author{
Ch. Ravi Kumar ${ }^{1 *}$, K. Padmaraju \\ ${ }^{I} M$ Tech (Ph.D) Sir, C R Reddy College of Engineering Eluru, Andhrapradesh India \\ ${ }^{2}$ Ph.D, Professor in ECE Department, JNTUK, Kakinada, Andhrapradesh India \\ *Corresponding Author E-mail: ${ }^{1}$ ravich230@gmail.com, ${ }^{2}$ prof.padmaraju@gmail.com
}

\begin{abstract}
The performance of soft decision decoding, whose for which the design is complex, is superior to the performance of hard decision decoding. In this paper, we propose a turbo product code with a bit flip algorithm to improve the performance of hard decision decoding. The performance of hard decision decoding is improved with low complexity using multidimensional turbo product codes. The reliability of decoding in a communication system to detect and correct errors is discussed .Maximum a posterior probability (MAP) decoding is employed to improve the hard decision performance of turbo product codes with multiple dimensions. Our results include comparisons of multiple dimensions-2D, 3D, 4D, and 5D - and the number of iterations in soft and hard decision decoding.
\end{abstract}

Keywords: MAP decoding, iterations, bit parity, product turbo code, soft decision, hard decision decoder, multiple dimensions

\section{Introduction}

Turbo codes comprise an effective coding scheme with a concatenation of codes; the performance of these codes is similar to the performance of the Shannon limit [2].Error correction and detection of different types of coding and performance improvement are detailed [3].The concept of turbo product codes, which is one of the simplest forms of a concatenation of codes, is introduced [4]. The concept of block codes in the form of product codes, which apply a separable MAP filter decoding technique using soft decision decoding ,is discussed [5]. To improve the performance and reliability of decoding techniques, the iterative decoding of block and convolution codes is performed [6]. The parity bits increase the error correcting capability to reduce the complexity of using single parity bits [7]. The performance of the Chase decoding algorithm for turbo codes using soft decision decoding is efficient. The concept of a hybrid decoder ,in which both hard decision decoding and soft decision decoding is employed, is introduced [10].

The concept of multidimensionality and a single parity for error performance is presented in this paper. This paper describes the performance difference between two decoding techniques: hard decision decoding and soft decision decoding. In this paper, the error correction capability increases as the number of iterations increase as it employs an iterative algorithm.

\section{Multidimensional Turbo Product Codes}

The turbo codes comprise a concatenation of two codes with the same component codes. The feature of turbo codes is to increase the coding gain by increasing the error performance. This paper employs the concatenation of two block codes with single parity.
The structure of product codes uses dual parity check operationsvertical and horizontal parity check operations - to detect the position of errors. The lengths of the codes are represented as (n, $\mathrm{k}, \mathrm{d} \min$ ), where $\mathrm{n}$ is the code word length , $\mathrm{k}$ represents $\mathrm{n}-1$ information and $\mathrm{d} \min$ is the minimum distance between two codes. The concatenation does not employ an interleaver, and serial concatenation is applied.

The multidimensional aspect of this paper is five dimensions: $5 \mathrm{Dmn}$. The structure of different dimensional turbo product codes, as shown in Fig. 1, is the 2Dmn turbo product code, which has the code components (n1,k1,dmin 1$)$ and $(\mathrm{n} 2, \mathrm{k} 2, \mathrm{dmin} 2)$ and lower complexity single horizontal and vertical parity

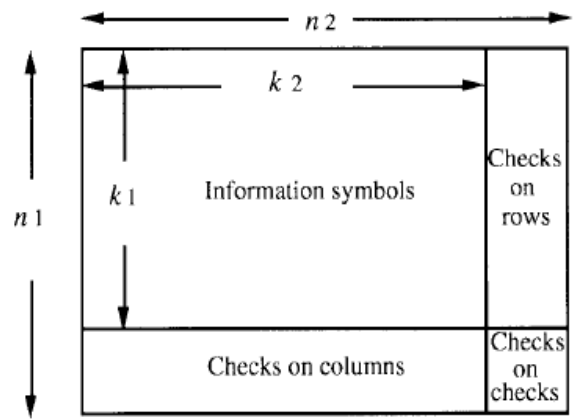

Fig. 1: 2Dm turbo product code

The construction of a 3Dmn product code has three code components in the three directions, $\mathrm{x}, \mathrm{y}$ and $\mathrm{z}$ : (n1,k1,dmin),(n2,k2,dmin2)and(n3,k3,dmin3).Horizontal and vertical parity bits ,such as px, py and pz, exist in each direction, as shown in Fig. 3. 


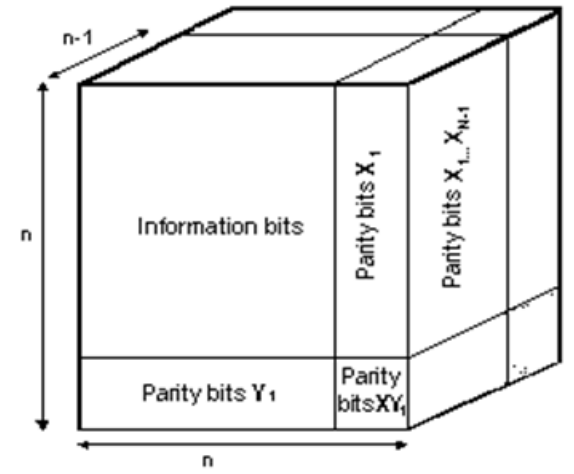

Fig. 2: 3Dm Turbo Product Codes

\section{Encoding of Turbo Product Codes}

The encoding concept of product codes is as follows:

1. Concatenation of block codes

2. Calculating parity bits in both directions

3. BPSK modulation under AWGN

4. Calculating code gain and $\mathrm{d}$ min values for each dimension The equations for calculating the code rate $\mathrm{R}$ and the $\mathrm{d}$ min are

$\mathrm{D} \min =2^{d}$ and $\mathrm{R}=\left(\frac{k}{n}\right)^{d}$

The data are represented in binary form as $\mathrm{d} 1, \mathrm{~d} 2 \ldots \ldots \mathrm{dn}-1$ and added to the parity bits in both directions; they are given as the code words $\mathrm{c} 1, \mathrm{c} 2, \mathrm{c} 3, \ldots \ldots . \mathrm{ck}$.

The modulated data on the BPSK modulation are given by $\mathrm{x} 1, \mathrm{x} 2, \mathrm{x} 3, \ldots \ldots . . \mathrm{xn}-1$ over AWGN channel noise with the noise components $\mathrm{n} 1, \mathrm{n} 2, \mathrm{n} 3, \ldots . . \mathrm{nn}-1$, which is represented as

$\mathrm{Yi}=\mathrm{xi}+\mathrm{ni}$

The AWGN probability density function for the condition of $\mathrm{xi}==1$ or -1 is given in Fig. 3. One side of Fig. 3shows $p(\mathrm{yi} \mid \mathrm{xi}=$ +1 ) for which $x i=+1$ was transmitted. The other side of Fig. 3 show $s p(y i \mid x i=-1)$ when $x i=-1$ was transmitted and the pdfs of their random variables.

The decoding technique in this paper is a hard decoding technique, in which the decoding of the bits or data is performed based on signs and the threshold values of the signals. The hard decision completely disregards the value of LLR and concentrates on the sign of the bit. To improve the reliability of hard decision decoding, we quantize each received signal and decode based on eight levels for three bits and 16 levels for four bits.

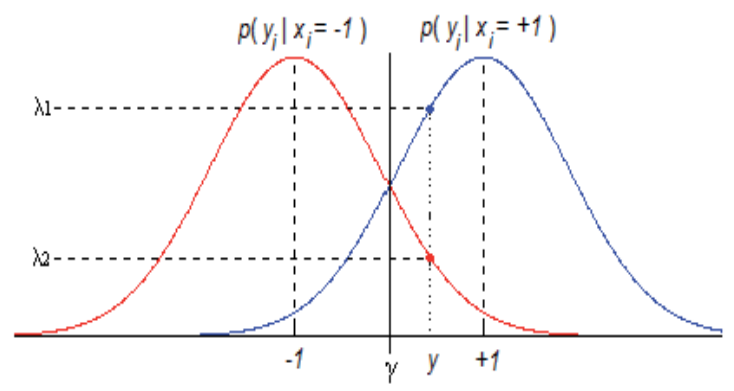

Fig. 3: AWGN likelihood functions

\section{Decoding of Turbo Product Codes}

The decoding of multidimensional turbo products starts by decoding the bits based on the quantized level values. The information bits that are received are represented as 0 and 1 . The syndrome will be calculated depending on the dimension of the received code. The syndrome calculation for $2 \mathrm{Dmn}$ data requires parity check operations to be performed in horizontal and vertical directions, as shown in Fig. 4.

\begin{tabular}{|l|l|l|}
\hline $\mathbf{1}$ & $\mathbf{0}$ & $\mathbf{1}$ \\
\hline $\mathbf{1}$ & $\mathbf{1}$ & $\mathbf{0}$ \\
\hline $\mathbf{0}$ & $\mathbf{1}$ & $\mathbf{1}$ \\
\hline
\end{tabular}

The data received after decoding are represented in the form of the 3Dmn product code.

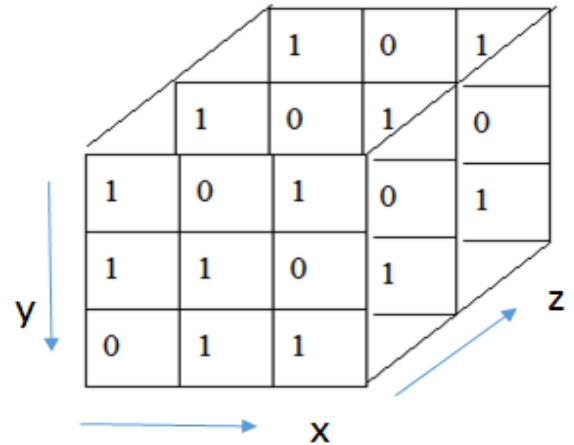

Fig. 4: $(3,2)^{3} 3 \mathrm{Dm}$ Turbo Product Code

The calculation of a syndrome in different directions is based on the dimension of the product code for the dimension applying a parity check operation in the yz direction, where as the y direction syndrome is calculated by a parity check operation in the $\mathrm{xz}$ direction

The three-dimensional syndrome value is given by

$$
\mathrm{Sm}=\mathrm{Sx}+\mathrm{Sy}+\mathrm{Sz}
$$

The decoding in this paper employs an iterative algorithm to achieve the reliable decoding; we increase the number of iterations. The summed syndrome value satisfies the threshold condition for the $\mathrm{i}^{\text {th }}$ iteration, in which the bit is flipped.

\section{A. Iterative Bit Flip}

The existing algorithm is a simple algorithm that is referred to as a bit flip. The condition is satisfied when the bit is flipped; this flipping operation continues until the ith iteration is completed. The problem in this bit flip algorithm is that if one bit is correct in a particular row and column and the condition is satisfied, it flips the bit without checking its reliability.

.The unnecessary flipping of bits increases the errors in the system. Low SNR values produce a large number of errors, whereas as high SNR values produce a small number of errors, which degrades the performance. This disadvantage of the existing algorithm yields an improved iterative algorithm

\section{B. Modified iterative Bit Flip algorithm}

This paper overcomes the disadvantages of the existing bit flip algorithm and proposes a weighted iterative bit flip algorithm to improve the error correcting capability. The iterative weighted bit flip algorithm checks the reliability of determining which bit needs to be flipped instead of flipping bits if the condition is satisfied. The algorithm calculates the error term and the reliability term. The algorithm calculates the weight of the bits; for each code, the high weight code bit is flipped.

The term for reliability is given as $R_{n m}$

$$
R_{n m}=\beta \frac{\left|y_{n}\right|}{\mid y_{m} \text { max } \mid}
$$

where $\mathrm{m}$ and $\mathrm{n}$ represent the rows and columns of the parity check 
operation based on the dimensions and

$y_{m}{ }^{\max }$ represent $\mathrm{s}$ the maximum magnitude value.

From the code word vectors from all other symbols

$\sum_{n: n \in N(m)} R_{m n}=1$

The error can be calculated as

$$
E_{n}=\sum_{m \in M(n)} \frac{\left(2 S_{m}-1\right)}{R_{m n}}
$$

where $s_{m}$ is the syndrome calculation,

$E_{n}$ is the error check sum calculation, and

$\beta$ is a normalized factor.

The proposed algorithm overcomes the problems of the bit flip algorithm and improves the performance of the error.

\section{Simulation Results}

A simulation was performed using the Mat lab tool. Decoding using multidimensional turbo product codes is described as follows: Multidimensional hard decoding improves the performance based on the BER shown in Fig. 5. This paper also deals about the soft decoding performance paper also addresses the soft decoding performance shown in Fig. 6 and Fig. 7.

Fig. 6 shows the performance of 4D hard and soft decision decoding in terms of BER.

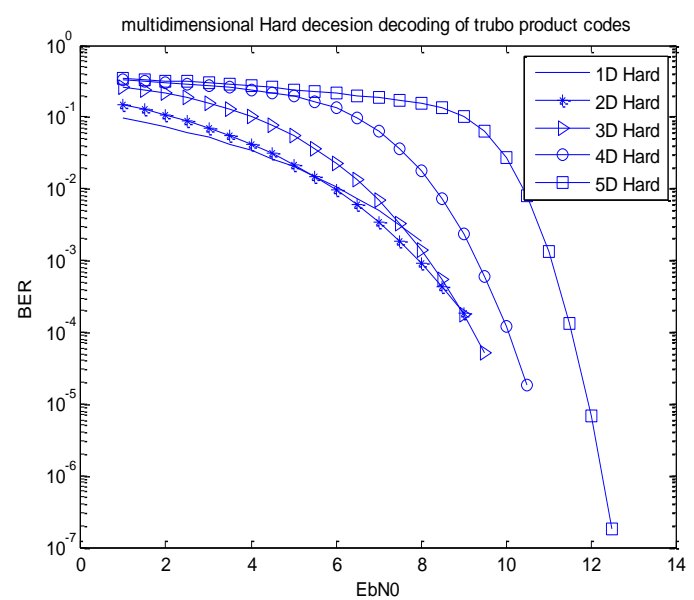

Fig. 5: Multidimensional turbo product codes for hard decision decoding

Fig. 7 shows the performance of 5D hard and soft decision decoding in terms of the BER. The performance is improved in 5D compared with the 4D codes. The performance gap is improved in terms of the BER.

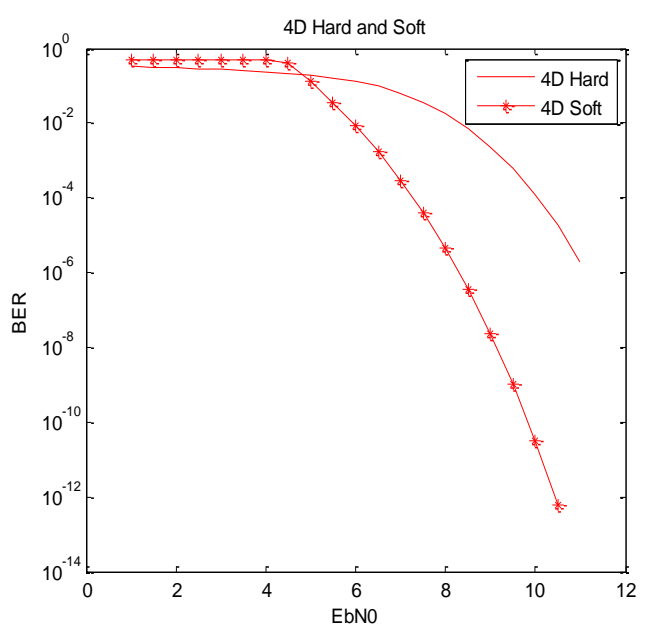

Fig. 6: 4D Hard and Soft Performance

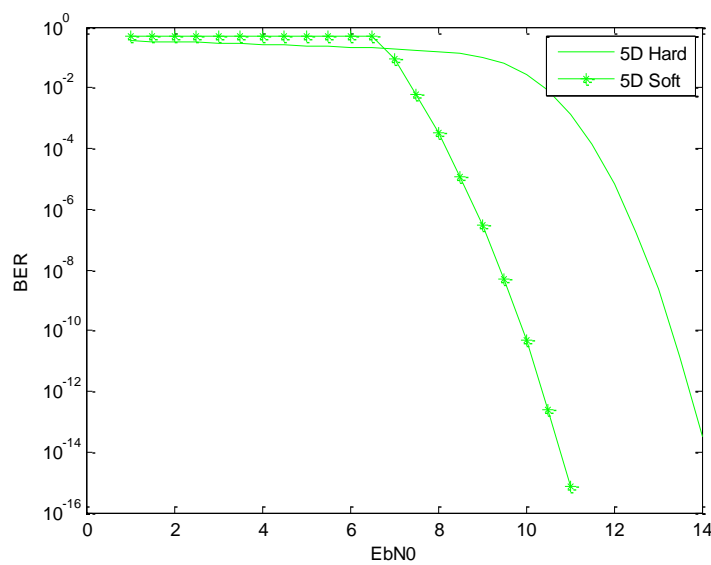

Fig. 7: 5D Hard and Soft Performance

This paper employs an iterative algorithm that shows the performance improvement as the number of iterations increase. Fig. 8 shows the improved performance as the iterations increase.

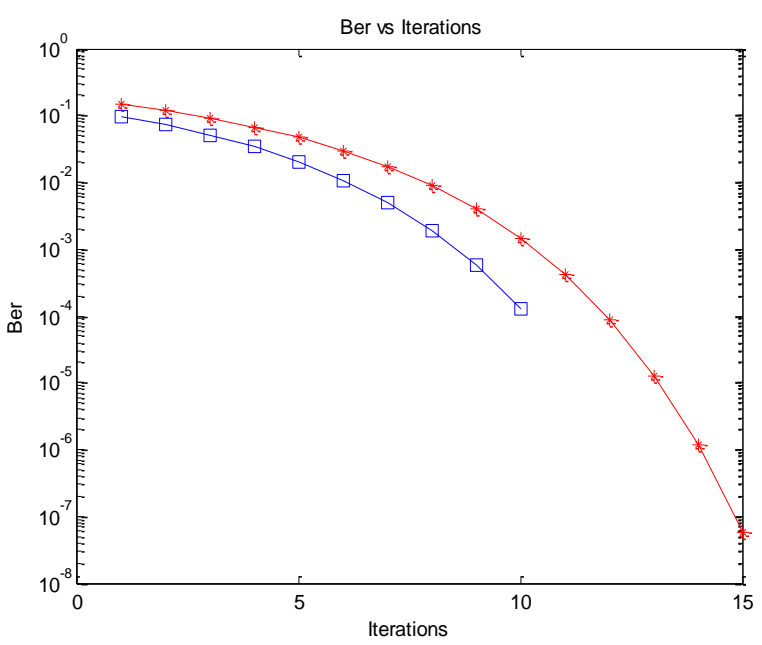

Fig.8: Performance based on iterations

\section{Conclusion}

The proposed iterative algorithm in this paper has achieved improved performance in hard decision decoding .Multidimensional turbo product codes and an iterative weighted flip algorithm can reduce the complexity. We use single parity to improve the error performance in terms of the BER. Low complexity hard decisions improve the reliability of decoding by considering the likelihood values in addition to the sign.

We justify that the algorithm performance has improved the decoding reliability and achieves a performance similar to the performance of soft decision decoding.

\section{References}

[1] Burr, "Turbo-codes: the ultimate error control codes" in Electronics \& Communication EngineeringJournal, vol. 13, pp. 155-165, IEEE, August 2001. ISSN: 0954-0695.

[2] C. Berrou, A.Glaxieux, and P. Thitimajhima, "Near Shannon limiterror-correcting coding and decoding: turbo codes (1)," in IEEE Int Conf. Communications ICC'93, vol. 2/3, May 1993, pp.1064-1071.

[3] P. Elias, "Error-free coding," IRE Trans. Inform. Theory, vol IT-4, pp. 29-37, Sept. 1954.

[4] R. M. Pyndiah, "Near-optimum decoding of product codes: block turbo codes," IEEE Trans. Commun, vol 46, pp. 1003-1010, Aug 1998.

[5] J. Lodge, R. Young, P. Hoeher, and J. Hagenauer, "Separable MAP "filters" for the de-coding of product and concatenated codes," in 
Proc. IEEE International Conference on Communications, Geneva, Switzerland, May 1993, pp. 1740-1745.

[6] J. Hagenauer, E. Offer, and L. Papke, "Iterative decoding of binary block and convolutional codes," IEEE Trans. Inform. Theory, vol 42, pp. 429-445, Mar 1996.

[7] D. M. Rankin and T. A. Gulliver, "Single Parity Check Product Codes", IEEE Trans, on Commun., vol. 49, no. 8, pp. 1354-1362, August 2001.

[8] C.Argon andW.McLaughlin, "An efficient Chase decoder for turbo product codes," IEEE Trans. Commun., vol. 52, no. 6, pp. 896-898, Jun. 2004

[9] L.Ying-Chang X.Changlong, and L.Wing Seng, "A low complexity decoding algorithm for turbo product codes", IEEE Wireless. Commun., vol. 7, no.1, pp. 43-47, Jan. 2008

[10] S.Zhiping, Z.Liang, F.Xiang, Y.Yang, HIHO Decoding Algorithms of Turbo Product Codes for High Rate Optical Communications, China Communications 2012, Vol. 9, pp. 114-123

[11] M. Baldi, F. Chiaraluce, G. Cancellieri, "Finite-Precision Analysis of Demappers and Decoders for LDPC-coded M-QAM Systems", IEEE Trans. on Broadcasting, Vol. 55, No. 2, pp. 239-250, June 200

[12] M. Hata., High-speed and robust error correcting code for future mobile communications of High-Dimensional Discrete Torus Knot,International symposium WPMC'01, Aalborg, Denmark, September 2001,

[13] A. J. Al-Dweik, S.L. Goff, B. S.Sharif, A Hybrid Decoder for BlockTurbo Codes, IEEE Transactions on Comm, vol. 57, No. 5, pp. 1229- 1232, May 2009

[14] A. J. Al-Dweik, B. S.Sharif, Non-Sequential Decoding Algorithm for Hard Iterative Turbo Product Codes, IEEE Transactions on Comm, vol.57, No. 6, pp. 1545-1549, June 2009

[15] H. Xu and F. Takawira, "A New Structure of Single Parity Check Product Codes", IEEE Africon, September 2004

[16] D. M. Rankin and T. A. Gulliver, "Single Parity Check Product Codes", IEEE Trans, on Commun., vol. 49, no. 8, pp. 1354-1362, August 2001.

[17] M. Rankin, T. A. Gulliver, "Parallel and Serial Concatenated Single Parity Check Product Codes", EURASIP Journal on Applied Signal Processing, pp. 775-783, January 2005 